\title{
BMJ Open Quality of patient, family, caregiver and public engagement in decision-making in healthcare systems: a scoping review protocol
}

\author{
Clayon Hamilton (D) , ${ }^{1,2,3}$ M Elizabeth Snow, ${ }^{4}$ Nancy Clark, ${ }^{5}$ Shannon Gibson, ${ }^{3}$ \\ Maryam Dehnadi, ${ }^{6}$ Michelle Lui, ${ }^{7}$ Andrew Koster, ${ }^{8}$ Janet McLean, ${ }^{9}$ Linda C Li (D) ${ }^{2}$
}

To cite: Hamilton C, Snow ME, Clark N, et al. Quality of patient, family, caregiver and public engagement in decision-making in healthcare systems: a scoping review protocol. BMJ Open 2019;9:e032788. doi:10.1136/ bmjopen-2019-032788

- Prepublication history and additional material for this paper are available online. To view these files, please visit the journal online (http://dx.doi. org/10.1136/bmjopen-2019032788).

Received 06 July 2019 Revised 09 0ctober 2019 Accepted 15 October 2019
Check for updates

(C) Author(s) (or their employer(s)) 2019. Re-use permitted under CC BY-NC. No commercial re-use. See rights and permissions. Published by BMJ.

For numbered affiliations see end of article.

Correspondence to Dr Clayon Hamilton; chamilton@arthritisresearch.ca

\section{ABSTRACT}

Introduction To advance person- and family-centred healthcare, government initiatives have supported the engagement of patients and family caregivers in decisionmaking in healthcare systems. There is, however, no consensus on how to define success for such initiatives. This scoping review aims to identify the key elements for defining the quality of patient and family caregiver engagement in decision-making across the engagement domains (individual, community/organisation, system) of British Columbia's healthcare system. We will use those elements to develop a conceptual evaluation framework. Methods and analysis This scoping review follows Arskey and 0'Malley's methodology. (1) The research question was identified through team discussions.

(2) Articles for data source will be identified using a librarian-informed search strategy for seven bibliographic databases as well as grey literature sources. (3) Selected articles will be relevant to the evaluation of patient and family caregiver engagement in healthcare systems. (4) Two researchers will independently extract data into predefined and emerging categories. (5) The researchers will reconcile and organise the identified elements. The research team's collective perspective will then refine the elements, and select, interpret and summarise the results. (6) Persons from key stakeholder groups will be consulted to refine the emergent conceptual framework.

Ethics and dissemination We will seek ethics approval for the stakeholder consultation. This study follows an integrated knowledge translation approach. The results will inform evaluation of the Patients as Partners Initiative of the British Columbia Ministry of Health, and will be disseminated as a scientific article, a research brief, and presentations at conferences and stakeholder meetings.

\section{INTRODUCTION}

Healthcare systems are starting to embrace a person- and family-centred healthcare approach to better meet the priorities of patients and the public. ${ }^{1-3}$ This approach positions patients, families and unpaid caregivers as partners engaged in healthcare decision-making and care processes. ${ }^{45}$
Strengths and limitations of this study

- A conceptual evaluation framework will be developed that covers all three domains of patient and family caregiver engagement in decision-making within healthcare systems.

- A comprehensive search strategy of electronic bibliographic sources published, and grey literature is being used to capture available evidence.

- This study employs an integrated knowledge translation approach involving a multi-stakeholder research team.

- The consultation of stakeholders from British Columbia will refine, contextualise and validate the content of the emergent conceptual evaluation framework, but may limit its direct applicability to international settings.

In British Columbia, the Patients as Partners Initiative was created in 2008 by the Ministry of Health to build capacity for, and strengthen the engagement of, patients, families, unpaid caregivers and the public in decision-making in the healthcare system. With the goal as an enabler for advancing person- and familycentred healthcare in the province, ${ }^{6-8}$ this initiative supports activities to include a patient and family caregiver voice, choice and representation in decision-making. The Ministry of Health's 2018 Patient, Family, Caregiver and Public Engagement Framework $^{8}$ depicts decision-making as occurring in three domains of engagement in the healthcare system: (1) the individual domain which comprises a person's and/or their family caregiver's direct involvement in a person's own care; (2) the community domain which denotes a person and their family caregiver taking part in activities related to healthcare programme and services and (3) the system domain which covers taking part in policy and strategic planning targeted at the healthcare 
system. ${ }^{8}$ The ultimate goal of the Patients as Partners Initiative is to support achieving the quadruple aims of optimal patient and provider experience, better health outcomes and better cost-effectiveness. ${ }^{9}{ }^{10}$ For simplicity, patient and family caregiver will be used to cover the many categories of individuals and groups served by the healthcare system, and who would be engaged as healthcare partners. We broadly define 'patients' as individuals served within a given context by a healthcare system from public health services of preventative care through to palliative care. Family is a biological or legal relative or an individual otherwise considered by a person to be family. A family caregiver refers to a 'family' member who provides unpaid care and support to a patient.

Tasked with evaluating the Patients as Partners Initiative, our research team has recognised the lack of a comprehensive framework for evaluating this type of policy-driven initiative. A good foundation for an evaluation framework is the Donabedian conceptual framework-a foundational tool that is widely accepted as a standard for guiding systematic evaluation of the quality of healthcare. ${ }^{11} 12$ Its three-dimensional approach of structure-process-outcome could be adopted and applied to conceptualise the quality of patient and family caregiver engagement in decision-making in healthcare systems. For the current study, structure comprises the settings in which engagement activities occurs, such as organisational structure, materials and human resources; process denotes the methods by which engagement occurs, such as the activities of patient and other stakeholders; and outcome is the effect of engagement activities, for example, improvements in patients' and family caregivers' knowledge, skills, behaviours and health status. ${ }^{11}$ The Donabedian framework thus provides overarching dimensions of a healthcare system within which to map the key elements on this topic of patient and family caregiver engagement in decision-making.

A recent systematic review by Dukhanin et al (2018) proposed a taxonomy of metrics for the evaluation of 'patient, public, consumer and community' engagement in decision-making at the organisation (ie, community) and system domains of engagement in healthcare systems. ${ }^{13}$ Their inductive qualitative analysis of 199 sources produced a taxonomy covering process and outcome metrics. ${ }^{13}$ A few commentaries on this taxonomy viewed it as useful for evaluation, and highlighted issues for improving its applicability. ${ }^{14-16}$ Notably, the taxonomy does not address engagement of individuals in their own care, although it is crucial for shared decision-making. ${ }^{1516}$ Second, it did not explicitly address structure metrics, such as institution and organisation characteristics, thus missing an important dimension of the Donabedian framework. ${ }^{11} 14-16$ One commentary explicitly noted that distinguishing structure metrics could have strengthened the taxonomy. ${ }^{14}$ Third, Dukhanin and colleagues reviewed only continuous systematic processes of engagement, to the exclusion of episodic and one-time engagement activities. $^{13}$
The quality of patient and family caregiver engagement is a value-laden concept that challenges finding consensus on elements of importance, reliable measures and methods for evaluation. ${ }^{17}$ Only limited evaluation tools within the community/organisation and system domains, such as the Public and Patient Engagement Evaluation Tool, ${ }^{18}$ are available with sparse evidence on their validity. Available frameworks define the levels and spectrum of patient and family caregiver engagement in healthcare decision-making. ${ }^{4}$ Other progress made in shaping the understanding of the quality of patient and family caregiver engagement in decision-making in healthcare systems include the Patient Health Engagement model ${ }^{5}$ and the Patient Health Engagement Scale ${ }^{5}$ which are both directed at the individual domain of engagement, and a systematic review of 11 evaluation tools focused on health system decision-making. ${ }^{19}$ The tools reported in that review seem to lack comprehensiveness and adequate validation. ${ }^{1419}$ Furthermore, we do not know the extent to which those tools cover the important elements of patient and family caregiver engagement since those elements have not been fully mapped out. Recently, Abelson and colleagues (2018) have reiterated the need for an evaluation framework for engagement at the organisation and system domains. ${ }^{20}$ Given the existing gap, there is a need for a conceptual evaluation framework for patient and family caregiver engagement in decision-making across all domains of engagement (individual, community/ organisation, system) in healthcare systems.

\section{Study rationale}

While patient and family caregiver engagement is touted as key for optimal and sustainable healthcare, ${ }^{21}{ }^{22}$ there is little evidence on whether patient and family caregiver engagement initiatives improve healthcare systems. A major barrier to developing this evidence base is a lack of consensus on how success in patient and family caregiver engagement should be defined (eg, what are the key elements and ideal outcomes of such engagement?). Therefore, a comprehensive framework, informed by the literature and perspectives of key stakeholders, is needed to provide a way for thinking about how patient and family caregiver engagement initiatives can be, and should be, evaluated. This framework would also be important for determining indicators to monitor and evaluate such initiatives. Furthermore, an evaluation framework would be helpful for establishing an agenda for research and policy on the quality of patient and family caregiver engagement in decision-making in healthcare systems.

\section{Study objective}

We aim to identify the key elements for defining the quality of patient and family caregiver engagement in decision-making across the three domains of engagement (individual, community/organisation and system) within the province of British Columbia healthcare system and use those elements to develop a conceptual evaluation framework. 
METHODS AND ANALYSIS

\section{Protocol design}

This study will be guided by Arksey and O'Malley's (2005) scoping review methodology enhanced by Levac et al (2010), and follow the standards of Preferred Reporting Items for Systematic Reviews and Meta-Analyse Extension for Scoping Reviews checklist. ${ }^{23-25}$ The methodology consists of six stages. They include (1) identifying the research question, (2) identifying relevant studies, (3) selecting studies, (4) charting the data, (5) collating, summarising and reporting results, and (6) consulting with stakeholders. ${ }^{23}{ }^{24}$ The final protocol will be prospectively registered with the Open Science Framework. ${ }^{25}$

\section{Stage 1: identify the research question}

The research question, proposed by the lead author $\mathrm{CBH}$, was refined through discussions within the research team, and fits the types of questions answered through scoping reviews. ${ }^{26}$ The primary question is 'What key elements define the quality of patient, family, caregiver, and public engagement in decision-making in healthcare systems for use in the evaluation of a provincial engagement initiative?'

\section{Stage 2: identify relevant studies}

Search terms were collaboratively determined by our research team. Our search strategy was informed by a university-based health science librarian with expertise in systematic literature reviews, a MEDLINE search filter for identifying patient and public engagement in health research, and the search strategy by Duhkanin et al (2018). ${ }^{1327}$ We will search seven electronic bibliographic databases: CINAHL (EBSCO), Cochrane Library (Ovid), EMBASE (Ovid), MEDLINE (Ovid), PsycINFO (EBSCO), Social Work Abstracts (EBSCO) and Web of Science from their inception to the search date of 23 April 2019 and update it on 14 June 2019. The proposed electronic search strategy for electronic databases is provided as online supplementary appendix A. The search strategy for MEDLINE was peer reviewed by the librarian using the Peer Review of Electronic Search Strategies) checklist. ${ }^{28}$

Our search will be broad because of the variety of terms used in this area, and the semi-organised and evolving nature of this body of literature. Search of the bibliographic databases will use a combination of four blocks of terms: (1) patient and family caregiver engagement, (2) decision-making, (3) evaluation and (4) healthcare system. For example, the first block pairs the terms for healthcare partners (eg, caregiver, community, consumer, family, patient, public, senior, stakeholder, user) and engagement (eg, advocate, activation, collaboration, consult, involve, participate, represent) and use indexing terms. Search terms will typically be used as both keywords in the title and/or abstract and subject headings as appropriate. No language or date limits will be set during the searches to capture articles translated to English from other languages and any foundational articles.
To capture all relevant articles, we will search reference lists of key articles and used Google Scholar to locate articles citing them. A targeted search of the grey literature will be conducted of relevant local, provincial, national and international organisations' websites and related health or scientific organisations for studies, reports and conference abstracts. Some grey literature sources will be selected from the list by Dukhanin et $a l,{ }^{13}$ the Canadian Agency for Drugs and Technologies in Health Grey Matters (a checklist of health-related grey literature sources from across the world), ${ }^{29}$ and the Canadian Evaluation Society's grey literature database. Finally, other literature will be identified by searching Google.ca, with a focus on the first 100 search hits for each set of search terms.

\section{Stage 3: select studies}

Retrieved articles will be transferred directly from a bibliographic database or Endnote (V.x7.8) reference management software to the Covidence software for screening, ${ }^{30}$ and duplicates of articles removed. The screening process will consist of two steps: (1) a title and abstract/summary and (2) full-text screening. For the first step, two researchers will independently screen the titles and abstracts of each retrieved article for inclusion against a set of minimum eligibility criteria using three rating options: no, yes and maybe. The selection process will be refined through periodic discussing between the researchers doing the screening. This is to ensure the eligibility criteria are robust enough to capture the articles that may relate to the evaluation of patient and family caregiver engagement in decision-making in healthcare systems. Articles deemed relevant by either or both reviewers (ie, combined yes or maybe ratings) will be included in the full-text review. In the second step, the full text of each article will be independently reviewed by two researchers to determine whether it meets the eligibility criteria using two rating options: exclude and include. Inter-rater agreement will be determined with the first 100 articles using simple agreement (the number of agreements divided by the number of comparisons) for step two screening, and then Cohen's $\kappa$ statistic calculated. $^{31} 32$ Disagreement about including any fulltext article will be settled by reviewing the article again and reconciling its eligibility through further discussions between the two researchers. When an agreement is not reached, a third researcher will be involved to obtained consensus.

\section{Eligibility criteria}

An article will be included when it (1) is available in the English Language, (2) describes patient and family caregiver engagement within healthcare systems and (3) provides useful information on aspects of patient and family caregiver engagement in decision-making to evaluate. 'Useful information' includes descriptions or definitions, information on relevance and information on relationships among aspects of patient and family 
caregiver engagement in decision-making. There will be no restrictions on the type of study design for research articles. An article will be excluded if the setting is (1) outside of the healthcare sector (eg, urban planning, forestry, transport), (2) specific to the research sector not directly related to healthcare, (3) specific to the education sector not directly related to healthcare and if (4) descriptions of engagement fall below the level of consult on the International Association for Public Participation (IAP2) spectrum of public participation. ${ }^{33}$ Engagement must be, therefore, at the level of consult, involve, collaborate or empower to be considered authentic engagement with patients and family caregivers. ${ }^{33}$ The IAP2 spectrum is consistent with the 'ladder of citizen participation' in the seminal work by Sherry Arnstein. ${ }^{33} 34$ Arnstein's ladder considers consultation and involvement to be tokenism because the citizen does not have power in decision-making, while the IAP2 considers them active levels of engagement because the public has influence but not power over decision-making. ${ }^{33} 34$ The eligibility criteria will undergo iterative refinement throughout the study as is common for scoping review. ${ }^{24}$

\section{Stage 4: data collection}

Full text of each selected article will be uploaded into NVivo qualitative data management and analysis software (QSR International Pty Ltd, Burlington, MA, USA). Two researchers will independently collect and tabulate the characteristics of each article, including its publication year, authors, article type (eg, original research, policy and guidelines), country of origin, healthcare setting and any other characteristics agreed on by the research team. Two researchers will use directed content analysis, ${ }^{35}$ a qualitative data analysis technique, to independently extract relevant information from the articles by coding them within six major categories: 'structure', 'process' and 'outcome' from the Donabedian framework, ${ }^{11}$ and 'individual (direct care)', 'community/organisation' and 'system' domains from the British Columbia Ministry of Health's engagement framework. ${ }^{8}$ Intercoder reliability between the two researchers for each of these six codes will be calculated as simple agreement using a subset of the articles at the start of the coding process. ${ }^{31} 36$ The minimum threshold for reliability will be $80 \%$. When this threshold is not reached for a code, the researchers will discuss the discrepancies, make any necessary refinements and independently apply the code to a new subset of articles. Even when the $80 \%$ agreement threshold is reached, negotiated agreement will be calculated where there are inconsistencies of coding between researchers. ${ }^{36}$ During negotiated agreement, the researchers will discuss the discrepancies to achieve a common understanding of the definition and use of each code. ${ }^{36}$ Once all of the articles are coded and any differences between researchers reconciled, these coded segments of each article will be open coded independently by the researchers to identify relevant elements for defining the quality of patient and family engagement in healthcare systems. A critical appraisal of the articles is not applicable given the nature of the data and aim of this study.

\section{Stage 5: data summary and synthesis of results}

Using a single file with all the data, the researchers who coded the articles will discuss the codes by comparing them towards combining comparable themes/concepts and naming and defining them as unique elements. These elements will be presented to, and discussed by, the research team to get a collective perspective on their names, definitions, appropriateness and acceptability. The elements will be suitably arranged to create an emergent conceptual evaluation framework. The research team will review and refine the emergent conceptual evaluation framework, noting any gaps.

\section{Stage 6: stakeholder consultation}

While stakeholder consultation is not mandatory in the Arksey and O'Malley's methodology, it will be conducted as recommended by Levac et al to increase the robustness, applicability, feasibility and acceptability of the conceptual framework. ${ }^{23}$ Adding credibility to the study, our team consists of the Patients as Partners Initiative lead, a programme evaluation specialist, a patient partner, a family caregiver partner and health services researchers. However, because this is an emerging and evolving area of practice and research, we will consult other persons from the key stakeholder groups (health system leaders and decisions-makers, managers and staff, healthcare providers and healthcare users) in British Columbia for their insights to supplement, confirm or refute and extend the emergent conceptual evaluation framework. The stakeholder consultation will be valuable to refine, contextualise and validate the framework for implementation in British Columbia. This study is embedded within the British Columbia Ministry of Health, and given its scope, time and fiscal constraints, we will not consult international stakeholders.

\section{Patient and public involvement}

A patient and a family caregiver have been members of our research team throughout the development of this scoping review protocol and will be actively involved in each stage of this study. The research team's process of working together is guided by the Patient Engagement in Research Framework, which outlines eight themes for ensuring meaningful patient engaged research. ${ }^{37}$ Starting at the initial conversation with the patient/family caregiver partners, the study lead $(\mathrm{CBH})$ sought to gather information that addressed each of the eight themes. He also worked with the patient/family caregiver partners to co-develop an understanding of the proposed project, the expected roles and time commitment. For example, discussions on the theme of 'convenience' helped the research team to decide on the best team meeting times, given each member's other activities and personal situations. ${ }^{37}$ The patient and family caregiver have contributed to shaping this protocol through team discussions. 
For example, they emphasised the unique role of family caregivers and that 'consult' can be authentic engagement for healthcare system decision-making. The specific contributions of the patient and family caregiver will be decided through team discussions as the study progresses through its stages.

\section{Ethics and dissemination}

A literature synthesis does not require research ethics board approval. Ethics approval will be sought, however, for the stakeholder consultation stage of this study. We will wait for the preliminary results from the literature synthesis before applying for research ethics board approval. This study follows an integrated knowledge translation approach, ${ }^{38}$ given the research team is a partnership between leadership of the Patients as Partners Initiative and other stakeholders. In addition to the patient perspectives to be captured from the literature and through stakeholder consultation, our patient/ family caregiver partners will contribute their perspectives throughout the entire research process and help to share findings with appropriate knowledge users. Furthermore, the family caregiver on our team represents a nonprofit organisation dedicated to advancing the priorities of family caregivers within the healthcare system. Each research team member will contribute to disseminating the results through conference presentations, a scientific article, a research brief and presentations at stakeholder meetings.

The conceptual evaluation framework on the quality of patient and family caregiver engagement in decisionmaking in healthcare systems will be a key step in the evaluation of the Patients as Partners Initiative. The results will inform evaluation of the Patients as Partners Initiative of the British Columbia Ministry of Health for quality improvement. We hope the framework will be applicable to other jurisdictions and provide guidance to determine the important domains and indicators for patient and family caregiver engagement initiatives in healthcare systems to advance person- and family-centred healthcare. A key reason the resulting framework could be applicable to other jurisdictions is the systematic approach being taken to gather evidence from the literature irrespective of jurisdictions. We plan to submit our findings for publication by April 2020.

\section{Author affiliations}

${ }^{1}$ Arthritis Research Centre of Canada, Richmond, British Columbia, Canada

${ }^{2}$ Department of Physical Therapy, The University of British Columbia, Vancouver, British Columbia, Canada

${ }^{3}$ Primary Care Division, British Columbia Ministry of Health, Victoria, British Columbia, Canada

${ }^{4}$ Program Evaluation, Centre for Health Evaluation \& Outcome Sciences, Vancouver, British Columbia, Canada

${ }^{5}$ Faculty of Human and Social Development, University of Victoria, Victoria, British Columbia, Canada

${ }^{6}$ Faculty of Health Sciences, Simon Fraser University, Burnaby, British Columbia, Canada

${ }^{7}$ School of Population \& Public Health, The University of British Columbia, Vancouver, British Columbia, Canada
${ }^{8}$ Patient partner, Victoria, British Columbia, Canada

${ }^{9}$ Family Caregivers of British Columbia, Victoria, British Columbia, Canada

Twitter Clayon Hamilton @Dr_Clayon and Linda C Li @LLi_1

Acknowledgements The authors thank Ursula Ellis, health sciences reference librarian at the University of British Columbia for help with developing the search strategy and for peer reviewing the search strategy using the PRESS checklist. Thanks to Shannon Holms, the former director of the Patients as Partners Initiation, for contributions to the initial conceptualisation of this study.

Contributors $\mathrm{CH}$ obtained funding, conceptualised the research and drafted this protocol. Each author (CH, MES, NC, SG, MD, ML, AK, JM and LCL) has made intellectual contribution to the development of this protocol and the acquisition of project funding. All authors (CH, MES, NC, SG, MD, ML, AK, JM and LCL) review and edited the protocol and provided input through team discussions and by email.

Funding This work is supported by SPOR Evidence Alliance through funding from the Canadian Institutes of Health Research (CIHR) under the Strategy for Patient Oriented-Research (SPOR) initiative grant number GSR-154442. CH is supported by a CIHR-MSFHR Health System Impact Fellowship from the BC Ministry of Health, Canadian Institute of Health Research, and Michael Smith Foundation of Health Research. CBH is also supported by a MSFHR Research Trainee award.

Competing interests SG is the manager of the Patients as Partners Initiative within the British Columbia Ministry of Health. JM is employed by Family Caregivers of British Columbia, a paid partner of the Patients as Partners Initiative, which is a non-profit organisation that provides support and advocates for family caregivers within British Columbia, Canada.

Patient consent for publication Not required.

Provenance and peer review Not commissioned; externally peer reviewed.

Open access This is an open access article distributed in accordance with the Creative Commons Attribution Non Commercial (CC BY-NC 4.0) license, which permits others to distribute, remix, adapt, build upon this work non-commercially, and license their derivative works on different terms, provided the original work is properly cited, appropriate credit is given, any changes made indicated, and the use is non-commercial. See: http://creativecommons.org/licenses/by-nc/4.0/.

\section{ORCID IDs}

Clayon Hamilton http://orcid.org/0000-0002-6852-3436

Linda C Li http://orcid.org/0000-0001-6280-0511

\section{REFERENCES}

1 Entwistle VA, Watt IS. Treating patients as persons: a capabilities approach to support delivery of person-centered care. Am J Bioeth 2013;13:29-39.

2 Sharma T, Bamford M, Dodman D. Person-Centred care: an overview of reviews. Contemp Nurse 2015;51:107-20.

3 McCormack B, Borg M, Cardiff S, et al. Person-centrednessthe'state' of the art. IPDJ 2015;5:1-15.

4 Carman KL, Dardess P, Maurer M, et al. Patient and family engagement: a framework for understanding the elements and developing interventions and policies. Health Aff 2013;32:223-31.

5 Graffigna G, Barello S. Spotlight on the patient health engagement model (PHE model): a psychosocial theory to understand people's meaningful engagement in their own health care. Patient Prefer Adherence 2018;12:1261-71.

6 McQuillen K, Davis C, Ho K, et al. Challenges in measuring patient participation: case studies from British Columbia's Patients as Partners initiative. J Participat Med 2013;5:e10.

7. Maika C, Harper C, Maria J. Patients as Partners. In: Patient engagement: catalyzing improvement and innovation in healthcare, 2016: 75-80. https://www.longwoods.com/content/24917/books/ patients-as-partnersChristine

8 Bar S, Grant K, Asuri S, et al. British Columbia Ministry of health patients as partners: a transformational approach. Healthc Manage Forum 2018;31:51-6.

9 Berwick DM, Nolan TW, Whittington J. The triple aim: care, health, and cost. Health Aff 2008;27:759-69.

10 Bodenheimer T, Sinsky C. From triple to quadruple aim: care of the patient requires care of the provider. Ann Fam Med 2014;12:573-6.

11 Donabedian A. The quality of care. How can it be assessed? JAMA 1988;260:1743-8. 
12 Hoenig $\mathrm{H}$, Lee J, Stineman M. Conceptual overview of frameworks for measuring quality in rehabilitation. Top Stroke Rehabil 2010;17:239-51.

13 Dukhanin V, Topazian R, DeCamp M. Metrics and evaluation tools for patient engagement in healthcare Organization- and system-level DecisionMaking: a systematic review. Int $\mathrm{J}$ Health Policy Manag 2018;7:889-903.

14 Boivin A. From Craft to Reflective Art and Science Comment on "Metrics and Evaluation Tools for Patient Engagement in Healthcare Organization- and System-Level Decision-Making: A Systematic Review". Int J Health Policy Manag 2019;8:124-7.

15 Berger Z. Metrics of Patient, Public, Consumer, and Community Engagement in Healthcare Systems: How Should We Define Engagement, What Are We Measuring, and Does It Matter for Patient Care? Comment on "Metrics and Evaluation Tools for Patient Engagement in Healthcare Organization- and System-Level Decision-Making: A Systematic Review". Int J Health Policy Manag 2018;8:49-50.

16 Sofaer S. Using the Taxonomy and the Metrics: What to Study When and Why Comment on "Metrics and Evaluation Tools for Patient Engagement in Healthcare Organization- and System-Level Decision-Making: A Systematic Review". Int J Health Policy Manag 2018:8:51-4.

17 Rosener JB. User-Oriented evaluation: a new way to view citizen participation. J Appl Behav Sci 1981;17:583-96.

18 Abelson J, Li K, Wilson G, et al. Supporting quality public and patient engagement in health system organizations: development and usability testing of the public and patient engagement evaluation tool. Health Expect 2016;19:817-27.

19 Boivin A, L'Espérance A, Gauvin F-P, et al. Patient and public engagement in research and health system decision making: a systematic review of evaluation tools. Health Expect 2018;21:1075-84.

20 Abelson J, Humphrey A, Syrowatka A, et al. Evaluating patient, family and public engagement in health services improvement and system redesign. Hcq 2018;21:31-7.

21 James JA. Patient engagement. Health Affairs Health Policy Brief 2013.

22 Fanjiang G, Grossman JH, Compton WD, et al. Building a better delivery system: a new engineering/health care partnership. National Academies Press, 2005.

23. Arksey H, O'Malley L. Scoping studies: towards a methodological framework. Int J Soc Res Methodol 2005;8:19-32.
24 Levac D, Colquhoun H, O'Brien KK. Scoping studies: advancing the methodology. Implementation Sci 2010;5.

25 Tricco AC, Lillie E, Zarin W, et al. PRISMA extension for scoping reviews (PRISMA-ScR): checklist and explanation. Ann Intern Med 2018;169:467-73.

26 Munn Z, Peters MDJ, Stern C, et al. Systematic review or scoping review? guidance for authors when choosing between a systematic or scoping review approach. BMC Med Res Methodol 2018;18:143.

27 Rogers M, Bethel A, Boddy K. Development and testing of a Medline search filter for identifying patient and public involvement in health research.. Health Info Libr J 2017;34:125-33.

28 McGowan J, Sampson M, Salzwedel DM, et al. PRESS Peer Review of Electronic Search Strategies: 2015 Guideline Statement. J Clin Epidemiol 2016;75:40-6.

29 Canadian Agency for Drugs and Technologies in Health (CADTH). Grey matters: a practical tool for searching health-related grey literature. Ottawa, ON Canada: Canadian Agency for Drugs and Technologies in Health (CADTH), 2018.

30. Covidence systematic review softwareMelborne, AustraliaCovidence, 2019Vertitas Health Innovation Ltd. Available: https://www. covidence.org/

31 Belur J, Tompson L, Thornton A, et al. Interrater reliability in systematic review methodology: exploring variation in Coder decision-making. Sociol Methods Res:0049124118799372.

32 Cohen J. A coefficient of agreement for nominal scales. Educ Psychol Meas 1960;20:37-46.

33 International Association for Public Participation. IAP2 spectrum of public participation, IAP2 International Federation, 2018. Available: https://cdn.ymaws.com/www.iap2.org/resource/resmgr/pillars/ Spectrum_8.5x11_Print.pdf

34 Arnstein SR. A ladder of citizen participation. J Am Inst Plann 1969;35:216-24.

35 Hsieh H-F, Shannon SE. Three approaches to qualitative content analysis. Qual Health Res 2005;15:1277-88.

36 Campbell JL, Quincy C, Osserman J, et al. Coding In-depth Semistructured Interviews:Problems of Unitization and Intercoder Reliability and Agreement. Sociol Methods Res 2013;42:294-320.

37 Hamilton CB, Hoens AM, Backman CL, et al. An empirically based conceptual framework for fostering meaningful patient engagement in research. Health Expect 2018;21:396-406.

38 Kothari A, McCutcheon C, Graham ID. Defining integrated knowledge translation and moving forward: a response to recent commentaries. Int J Health Policy Manag 2017;6:299-300. 